\title{
Расширенная модель Холстейна-Хаббарда для эпитаксиального графена на металле
}

\author{
(C) С.Ю. Давыдов \\ Физико-технический институт им. А.Ф. Иоффре Российской академии наук, \\ 194021 Санкт-Петербург, Россия \\ Санкт-Петербургский национальный исследовательский университет \\ иноормационных технологий, механики и оптики, \\ 197101 Санкт-Петербург, Россия \\ E-mail: Sergei_Davydov@mail.ru
}

(Получена 15 марта 2017 г. Принята к печати 30 марта 2017 г.)

\begin{abstract}
Предложена модель, объединяющая расширенную модель Хаббарда, включающую внутриатомное и межатомное кулоновское отталкивание, и модель Холстейна, описывающую взаимодействие зонного электрона с эйнштейновским фононом. Рассмотрены три области фазовой диаграммы: волны спиновой и зарядовой плотностей и однородное по спину и заряду состояние. Численные оценки для подложек $\mathrm{Rh}, \mathrm{Ir}$ и Pt показывают, что кулоновское взаимодействие играет ведущую роль, создавая возможность переходов из однородного состояния в состояния волн спиновой и зарядовой плотности состояний.
\end{abstract}

DOI: 10.21883/FTP.2018.02.45449.8581

\section{1. Введение}

Опубликованные более полувека назад статьи Холстейна [1] и Хаббарда [2] породили модель ХолстейнаХаббарда (ННМ), где совместно учитываются электронфононное $(e-p h)$ и электрон-электронное $(e-e)$ взаимодействия [3-11]. Модель Холстейна-Хаббарда применяется к описанию широкого набора физических состояний и структур: сверхпроводимости (в том числе, высокотемпературной) $[3,4,7]$, волн спиновой и зарядовой плотностей (ВСП и ВЗП соответственно) [5,7,10], поляронов и биполяронов $[3,4,6,8-10], 3 \mathrm{D}-[1,2,7,9], 2 \mathrm{D}-[6,10]$ и $1 \mathrm{D}$-решеток $[3,5,8,11]$, а также кластеров [3,4]. Общие математические особенности НHМ обсуждались в работе [12].

Следует подчеркнуть, что во всех цитированных работах (кроме работы [12]) рассматривается только внутриатомное (одноцентровое) кулоновское отталкивание электронов с противоположными спинами $U$ (модель Хаббарда), а межатомным (межцентровым) отталкиванием электронов $G$ пренебрегается. В данной работе мы восполним этот пробел, воспользовавшись результатами работы [13], где электронное состояние свободного (СГ) и эпитаксиального (ЭГ) графена рассматривалось в рамках расширенной модели Хаббарда (ЕНМ), включающей как $U$, так и $G$. Объединяя модель ЕНМ с теорией Холстейна, получим расширенную моделью Холстейна-Хаббарда (ЕННМ). В рамках этой модели мы рассмотрим как СГ, так и ЭГ. В первом случае учтем длинноволновые оптические колебания атомов углерода в плоскости графена, во втором - добавим колебания адатомов углерода относительно подложки.

\section{2. Свободный графен}

Представим гамильтониан свободного графена $H_{\mathrm{FG}}$ в виде суммы электрон-электронного $H_{e-e}$ и электрон- фононного $H_{e-p h}$ гамильтонианов:

$$
H_{\mathrm{FG}}=H_{e-e}+H_{e-p h} .
$$

В соответствии с ЕНM, гамильтониан $H_{e-e}$ дается выражением

$$
\begin{aligned}
H_{e-e}= & \varepsilon_{0} \sum_{i} \hat{n}_{i}+U \sum_{i} \hat{n}_{i \uparrow} \hat{n}_{i \downarrow}+G \sum_{\langle i, j\rangle} \hat{n}_{i} \hat{n}_{j} \\
& -t \sum_{\langle i, j\rangle, \sigma}\left(a_{i \sigma}^{+} a_{j \sigma}+a_{j \sigma}^{+} a_{i \sigma}\right),
\end{aligned}
$$

где $\varepsilon_{0}$ - энергия состояния $\left|p_{z}\right\rangle$ изолированного атома углерода, $t$ - энергия перехода (перескока) электрона между ближайшими соседями (б.с.) в графене, $\hat{n}_{i \sigma}=a_{i \sigma}^{+} a_{i \sigma}-$ оператор числа заполнения $\left|p_{z}\right\rangle$-состояния $i$-го узла решетки графена $|i, \sigma\rangle$, $\sigma=(\uparrow, \downarrow)-$ проекция спина, $\hat{n}_{i}=\sum_{\sigma} \hat{n}_{i \sigma} ; a_{i \sigma}^{+}\left(a_{i \sigma}\right)-$ оператор рождения (уничтожения) электрона графена в состоянии $|i, \sigma\rangle,\langle i, j\rangle$ означает суммирование по б.с. в графене [13].

Учтем теперь колебания атомов углерода в плоскости графена по схеме работ $[14,15]^{1}$ (аналогичный подход использован в $[3,6])$, представив гамильтониан в виде

$$
H_{e-p h}=\sum_{i}\left(\frac{1}{2} M \dot{x}_{i}^{2}+\frac{1}{2} M \omega_{\|}^{2}\left(\delta x_{i}\right)^{2}+w_{\|} \delta x_{i} \hat{n}_{i},\right)
$$

где $w_{\|}$и $\omega_{\|}$- деформационная константа $e-p h$-взаимодействия и частота длинноволнового оптического фонона для колебаний в плоскости графена, $\delta x_{i}-$ смещение атома в узле $x_{i}, M$ - масса атома углерода. В гамильтониане (3) мы опустили $t$-взаимодействие, входящее в стандартную модель Холстейна [1], так как оно уже учтено в выражении (2).

\footnotetext{
${ }^{1}$ В работе [15] в формуле (2) допущена опечатка: второе слагаемое в правой части должно иметь вид $M \omega_{0}^{2}\left(z-z_{0}\right)^{2} / 2$.
} 
Применяя к гамильтониану $H_{\mathrm{HG}}$ теорему $\partial\left\langle H_{\mathrm{FG}}\right\rangle / \partial \delta x_{i}=\left\langle\partial H_{\mathrm{FG}} / \partial \delta x_{i}\right\rangle \quad[14,15], \quad$ получим $\delta x_{i}=$ $=-\left(w_{\|} / M \omega_{\|}^{2}\right) n_{i}$. Рассматривая гамильтониан $H_{e-e}$ в приближении Хартри-Фока, вместо $\varepsilon_{0}$ можем записать перенормированные значения $\varepsilon_{i \sigma}=\varepsilon_{0}+U n_{i-\sigma}$ $+z G n_{j}-\lambda_{\|} n_{i}, \quad$ где константа $e-p h$-взаимодействия $\lambda_{\|}=w_{\|}^{2} / M \omega_{\|}^{2}, n_{j}-$ число заполнения ближайшего к узлу $i$ соседа $j, z=3-$ число б. с. в графене. Переходя к явному учету подрешеток $(\alpha=A, B)$, запишем уровни энергии $\varepsilon_{\alpha \sigma}$ в виде $\varepsilon_{A(B) \uparrow, \downarrow}=\varepsilon_{0}+U n_{A(B) \downarrow, \uparrow}+z G n_{A(B)}$ $-\lambda_{\|} n_{A(B)}$. Введем обозначения: $2 a=n_{A \uparrow}+n_{A \downarrow}+n_{B \uparrow}$ $+n_{B \downarrow}, 2 b=n_{A \uparrow}-n_{A \downarrow}+n_{B \uparrow}-n_{B \downarrow}, 2 c=n_{A \uparrow}+n_{A \downarrow}-n_{B \uparrow}$ $-n_{B \downarrow}, \quad 2 d=n_{A \uparrow}-n_{A \downarrow}-n_{B \uparrow}+n_{B \downarrow}$, где $2 b, \quad 2 c \quad$ и $2 d$ являются параметрами порядка, описывающими соответственно намагниченность, ВЗП- и ВСПсостояния двухатомной элементарной ячейки графена, содержащей $2 a$ электронов. Числа заполнения атомов подрешеток при этом есть $n_{A \uparrow, \downarrow}=(a \pm b+c \pm d) / 2$, $n_{B \uparrow, \downarrow}=(a \pm b-c \mp d) / 2, \quad$ откуда $\quad n_{A(B)}=a \pm c, \quad \mathrm{a}$ энергии уровней равны

$$
\begin{aligned}
& \varepsilon_{A \uparrow, \downarrow}=\varepsilon_{0}+U(a \mp b+c \mp d) / 2+z G(a-c)-\lambda_{\|}(a+c), \\
& \varepsilon_{B \uparrow, \downarrow}=\varepsilon_{0}+U(a \mp b-c \pm d) / 2+z G(a+c)-\lambda_{\|}(a-c) .
\end{aligned}
$$

Введем энергию $\varepsilon^{\prime}=\varepsilon_{0}+a\left(U / 2+z G-\lambda_{\|}\right)$. Легко показать, что предположив $\varepsilon^{\prime}=\varepsilon_{\mathrm{F}}=0$, где $\varepsilon_{\mathrm{F}}-$ уровень Ферми, получим недопированный графен с $a=1$ (валентная зона заполнена, зона проводимости пуста) [13]. В дальнейшем будем рассматривать нулевую намагниченность элементарной ячейки, предполагая $b=0$. Тогда вместо (4) имеем $\varepsilon_{A \uparrow, \downarrow}=U(c \mp d) / 2-c\left(z G+\lambda_{\|}\right)$ и $\varepsilon_{B \uparrow, \downarrow}=-U(c \mp d) / 2+c\left(z G+\lambda_{\|}\right)$. При этом удобно ввести эффективное межатомное отталкивание

$$
G^{\prime}=G+\lambda_{\|} / z .
$$

Отметим, что в ННМ (см., например работы $[3,5,7])$, где $G=0$, роль $e-p h$-взаимодействия сводят к перенормировке внутриатомного кулоновского отталкивания: вместо $U$ появляется $U_{\text {eff }}<U$. В рассматриваемой нами задаче при $a=1$ и $b=0$ чисто математически удобнее ввести взаимодействие $G^{\prime}$. При этом все выводы разд. 3 работы [13] остаются в силе при замене $G$ на $G^{\prime}$.

\section{3. Эпитаксиальный графен}

Рассмотрим изолированный адатом углерода, находящийся в узле $i$. Гамильтониан $e-e$-взаимодействия для такого адатома есть

$$
\begin{aligned}
h_{e-e, i}= & \sum_{\mathbf{q}, \sigma} \varepsilon_{\operatorname{sub}}(\mathbf{q}) \hat{n}_{\mathbf{q}, \sigma}+\varepsilon_{0} \hat{n}_{i}+U \hat{n}_{i \uparrow \hat{n}_{i \downarrow}} \\
& +V \sum_{\mathbf{q}, i, \sigma}\left(c_{\mathbf{q} \sigma}^{+} a_{i \sigma}+h . c\right),
\end{aligned}
$$

где $\varepsilon_{\text {sub }}(\mathbf{q})-$ закон дисперсии электронов подложки, $\hat{n}_{\mathbf{q} \sigma}=c_{\mathbf{q} \sigma}^{+} c_{\mathbf{q} \sigma}-$ оператор числа заполнения состояния $|\mathbf{q}, \sigma\rangle$ с волновым вектором q и проекцией спина $\sigma$, $c_{\mathbf{q} \sigma}^{+}\left(c_{\mathbf{q} \sigma}\right)-$ соответствующий оператор рождения (уничтожения) электрона подложки, $V$ - матричный элемент взаимодействия, спаривающий состояния $|\mathbf{q}, \sigma\rangle$ и $|i, \sigma\rangle$. Учтем теперь локальное колебание изолированного адатома углерода вдоль оси $Z$, перпендикулярной поверхности подложки, определив соответствующий гамильтониан как

$$
h_{e-p h, i}^{\perp}=\frac{1}{2} M \dot{a}_{\perp i}^{2}+\frac{1}{2} \omega_{\perp}^{2}\left(\delta a_{\perp i}\right)^{2}+w_{\perp} \delta a_{\perp i} \hat{n}_{i},
$$

где $w_{\perp}-$ деформационная константа $e-p h$-взаимодействия для колебания адатома относительно подложки с частотой $\omega_{\perp}$, соответствующая изменению длины $\delta a_{\perp i}$ адсорбционной связи $a_{\perp i}$. Здесь и в дальнейшем мы считаем атомы металлической подложки неподвижными на том основании, что атомы переходных металлов много тяжелее атома углерода.

Можно показать, что $\delta a_{\perp i}=-\left(w_{\perp} / M \omega_{\perp}^{2}\right) n_{i}$. В этом случае имеем $\varepsilon_{A(B) \uparrow, \downarrow}=\varepsilon_{0}+U n_{A(B) \downarrow, \uparrow}+z G^{\prime} n_{B(A)}-\lambda_{\perp} n_{A(B)}$, где $\lambda_{\perp}=w_{\perp}^{2} / M \omega_{\perp}^{2}$. Полагая $b=0$ и $\varepsilon^{\prime \prime}=\varepsilon_{0}+a(U / 2$ $\left.+z G^{*}\right)=0$ (т.е. $\left.a=1\right)$, получим $\varepsilon_{A \uparrow, \downarrow}=U(c \mp d) / 2$ $-c z G^{*}$ и $\varepsilon_{B \uparrow, \downarrow}=-U(c \mp d) / 2+c z G^{*}$, где

$$
G^{*}=G+\left(\lambda_{\|}+\lambda_{\perp}\right) / z .
$$

По аналогии с данными работы [13], энергия системы может быть представлена в виде

$$
\begin{aligned}
E= & \sum_{\alpha \sigma} \varepsilon_{\alpha \sigma} \hat{n}_{\alpha \sigma}-U \sum_{\alpha} n_{\alpha \uparrow} n_{\alpha \downarrow}-z G n_{A} n_{B} \\
& +\sum_{\alpha, \sigma} \int_{-\infty}^{\varepsilon_{\mathrm{F}}-\varepsilon_{\alpha \sigma}} \Omega_{\sigma} \rho_{\alpha}^{\sigma}\left(\Omega_{\sigma}\right) d \Omega_{\sigma}+E_{\mathrm{sub}}+E_{\mathrm{ph}} .
\end{aligned}
$$

Здесь $\quad \Omega_{\sigma}=\varepsilon-\varepsilon_{0} \mp \Delta_{\sigma}^{*}, \quad \varepsilon_{\sigma}=\left(\tilde{\varepsilon}_{A \sigma}+\tilde{\varepsilon}_{B \sigma}\right) / 2, \quad \Delta_{\sigma}^{*}=$ $=\left(\tilde{\sigma}_{A \sigma}-\tilde{\varepsilon}_{B \sigma}\right) / 2, \tilde{\varepsilon}_{\alpha \sigma}=\varepsilon_{\alpha \sigma}+\Lambda(\varepsilon)$, где $\Lambda(\varepsilon)-$ гибридизационный сдвиг энергетических уровней за счет взаимодействия с подложкой, $\rho_{\alpha}^{\sigma}\left(\Omega_{\sigma}\right)-$ плотность состояний (на атом) $\alpha$-подрешетки ЭГ, $\varepsilon-$ энергетическая переменная; $E_{\mathrm{sub}}-$ вклад субстрата в энергию системы, $E_{\mathrm{ph}}=E_{\mathrm{ph}}^{\|}+E_{\mathrm{ph}}^{\perp}$, где $E_{\mathrm{ph}}^{\|} \quad$ дается первыми двумя слагаемыми в правой части (3), $E_{\mathrm{ph}}^{\perp}=(M / 2) \sum_{i}\left[\dot{a}_{\perp i}^{2}+\omega_{\perp}^{2}\left(\delta a_{\perp i}\right)^{2}\right]$. На этом общее построение ЕННМ для ЭГ можно считать законченным.

Далее мы рассмотрим металлическую подложку $(d$-металл), предполагая плотность состояний $\rho_{\text {sub }}(\varepsilon)=\rho_{m}=$ const, так что полуширина квазиуровня адатома углерода принимает вид константы $\Gamma_{m}=\pi V^{2} \rho_{m}$, а гибридизационный сдвиг квазиуровня $\Lambda_{m}(\varepsilon)$ обращается в нуль $[13,14]$.

Положим для простоты $t=0$. Легко показать, что в общем случае приходим к уравнениям (22), а при $a=1$, $b=0$ и $\varepsilon^{\prime \prime}=\varepsilon_{\mathrm{F}}=0-$ к уравнениям $(23)$ из работы [13], где, однако, $G$ нужно заменить на $G^{*}$. Отсюда следует, что фазовая диаграмма, изображенная на рис. 2 в работе [13], остается в силе при замене $g=G / \pi \Gamma_{m}$ на 
$g^{*}=G^{*} / \pi \Gamma_{m}>g$. Таким образом, $e-p h$-взаимодействие облегчает переход в ВЗП-состояние.

Учтем теперь прямой обмен между атомами ЭГ $(t \neq 0)$, для чего рассмотрим кластер из шести адатомов углерода (рис. 3 из работы [13]). Предполагая, что $a=1, b=0, \varepsilon_{\mathrm{F}}=\varepsilon^{\prime \prime}=0$, получим аналог системы уравнений (26) из работы [13], где $G$ следует заменить на $G^{*}$. В этом случае система конечна и следует говорить не о волнах, а о неоднородных зарядовой и спиновой плотностях (НЗП и НСП). Легко показать, что состояния НЗП и НСП реализуются при выполнении следующих неравенств:

$$
\begin{gathered}
2 z G^{*}-U>\pi \Gamma_{m} \eta, \\
U>\pi \Gamma_{m} \eta,
\end{gathered}
$$

где $\eta=\left(t \sqrt{z} / \Gamma_{m}\right)\left(\arctan \left(t \sqrt{z} / \Gamma_{m}\right)\right)^{-1}>1$. Отсюда следует, что фазовая диаграмма, изображенная на рис. 4 в работе [13], остается справедливой при замене $g=G / \pi \Gamma_{m}$ на $g^{*}=G^{*} / \pi \Gamma_{m}$. Можно показать также, что учет допирования в настоящем случае дает результаты, аналогичные полученным в п. 4.3 работы [13] с заменой $G$ на $G^{*}$.

\section{4. Численные оценки}

Начнем с оценок для СГ, полагая $w_{\|}=-\partial V_{2 \|} / \partial a_{\|}$, где $V_{2 \|}=t=2.8$ эВ [16] - матричный элемент $\pi$-связи б.с. в графене, $a_{\|}=1.42 \AA-$ расстояние между б.с. в плоскости графена. Учитывая, что согласно методу связывающих орбиталей (МСО) Харрисона $t \propto a_{\|}^{-2}$ [17], получаем $w_{\|}=2 t / a_{\|} \approx 3.9$ эВ/А. Так как $\hbar \omega_{\|} \approx 0.20$ эВ [18], имеем $\lambda_{\|}=w^{2} / M \omega_{\|}^{2} \approx 0.13$ эВ.

Некоторым обоснованием применимости МСО для оценки константы $\lambda_{\|}$служит следующее обстоятельство. Значение $\hbar \omega \approx 0.20$ эВ соответствует силовой константе $k_{\|}=M \omega_{\|}^{2} \approx 116$ эВ $/ \AA^{2}$. Согласно данным работы [19], $k_{\|}=3\left(k_{0 \|}+6 k_{1 \|}\right)$, где $k_{0 \|},\left(k_{1 \|}\right)$ - константа центрального (нецентрального) взаимодействия б.с. в графене. В соответствии с данными работы [20] (при $\left.V_{1}=0\right)$ между центральной и нецентральной константами имеем место соотношение $k_{1 \|}=0.22 k_{0 \|}$, так что $k_{0 \|} \approx k_{\|} / 7 \approx 17$ эВ/ $\AA^{2}$. С другой стороны, прямой расчет дает $k_{0 \|} \approx 4 V_{2 \|} / a_{\|}^{2} \approx 24$ эВ/ $\AA^{2}$. Мы считаем такое соответствие приемлемым для порядковых оценок.

Перейдем теперь к ЭГ на металлической подложке. Для оценки констант $e-p h$-взаимодействия воспользуемся моделью двухатомной поверхностной молекулы [14], рассматривая $\sigma$-связь $\left|p_{z}\right\rangle$-орбитали углерода и $|d\rangle$-орбитали металлического атома, описываемую матричным элементом $V_{2 \perp}$. Предположим, что $w_{\perp}=-\partial V_{2 \perp} / \partial a_{\perp}, \quad$ a $\quad V_{2 \perp}=V_{p d \sigma}=(3 \sqrt{15} / 2 \pi) \hbar^{2}$ $\times\left(r_{d}^{3} r_{p}\right)^{1 / 2} / m_{0} a_{\perp}^{4}[21]$, где $r_{p(d)}-$ радиус $p(d)$-состояния, $m_{0}-$ масса свободного электрона. Тогда $w_{\perp}=4 V_{2 \perp} / a_{\perp}$. В соответствии с МСО для ковалентной связи полагаем $a_{\perp}=r_{\mathrm{C}}+r_{\mathrm{M}}$, где $r=0.77 \AA-$ атомный радиус углерода, $r_{M}-$ атомный радиус металла. По данным справочника [22] для центральных элементов $d$-рядов получаем $a_{\perp} \approx 2 \AA$, так что $V_{2 \perp} \approx 2$ эВ и

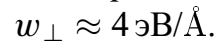

Для вычисления $k_{0 \perp}=\left(\partial^{2} E_{d} / \partial a_{\perp}^{2}\right)_{0}$, где индекс 0 отвечает положению равновесия, зададим энергию отталкивания в виде $V_{\text {rep }}=A_{\text {rep }}\left(r_{d} / a_{\perp}\right)^{8}$ [23], так что энергия двухэлектронной связи равна $E_{d}=2\left(-V_{2 \perp}+V_{\text {rep }}\right)$.

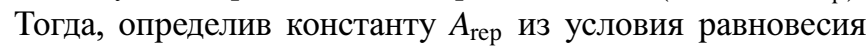
$\left(\partial E_{d} / \partial a_{\perp}\right)_{0}=0$, легко показать, что $k_{0 \perp}=32 V_{2 \perp} / a_{\perp}^{2}$, откуда $\lambda_{\perp}=V_{2 \perp} / 2$. Таким образом, $k_{0 \perp} \approx 16$ эВ $/ \AA^{2}$ и $\lambda_{1} \approx 1$ эВ.

Сделанные оценки относятся к сильно (ковалентно) связанному с металлом ЭГ и отвечают колебанию плоского листа графена как целого относительно подложки. В случае изгибных колебаний листа (OZ-колебания) ситуация обстоит сложнее: при этом изменяется не только длина адсорбционной связи $a_{\perp}$, за деформацию которой отвечает константа $k_{0 \perp}$, но и расстояние между б.с. в плоскости графена $a_{\|}$, что описывается константой $k_{0 \|}$ (по поводу связи продольных и поперечных колебаний в ЭГ см. работу [24]). В этом случае простая модель поверхностной молекулы уже не работает. Для OZколебания СГ имеем $\hbar \omega_{\perp} \approx 0.10$ эВ [18], что отвечает силовой константе $k_{0 Z} \approx 29$ эВ/ $\AA^{2}$, что качественно не отличается от полученного выше значения. Поэтому в дальнейшем будем считать значение $\lambda_{\perp} \sim 1$ эВ оценкой по максимуму.

Анализ экспериментальных данных в работах $[25,26]$ привел к выводу, что расстояния между графеновым слоем и подложками $\operatorname{Pt}(111)$ и $\operatorname{Ir}(111)$ равны соответственно $3.70 \AA[25]$ и $3.80 \AA$ [26] и весьма близки к постоянным решетки платины и иридия, равным соответственно 3.92 и $3.84 \AA$ [27]. Таким расстояниям $a_{\perp}$ отвечает уже не ковалентное, а ван-дер-ваальсово взаимодействие графен-подложка. Для описания такой связи используем потенциал Леннарда-Джонса $V_{\mathrm{LD}}\left(a_{\perp}\right)=4 D\left[\left(\bar{\sigma} / a_{\perp}\right)^{12}-\left(\bar{\sigma} / a_{\perp}\right)^{6}\right]$, где равновесное значение $a_{\perp}$ равно $\sqrt[6]{2} \bar{\sigma}$ и $D-$ величина энергии связи. Деформационную константу $w_{\perp}$ определим как производную по $a_{\perp}$ от ван-дер-ваальсова притяжения (второе слагаемое в квадратных скобках), что дает $w_{\perp}=12 D / a_{\perp}$. Тогда $k_{0 \perp}=72 D / a_{\perp}^{2}$, откуда получаем $\lambda_{\perp}=2 D$. За длину адсорбционной связи $a_{\perp}$ примем среднее значение постоянных решетки $\mathrm{Pt}, \mathrm{Ir}$ и $\mathrm{Rh}$, равное $3.85 \AA$, откуда получим $w_{\perp} \approx 3$ эВ $/ \AA$, $k_{0 \perp} \approx 5 D$ эВ $/ \AA^{2}$.

Теперь оценим значение параметра $\Gamma_{m}=\pi V^{2} \rho_{m}$ для $\mathrm{Rh}$, Ir и Pt. Ширины $d$-зон $W_{d}$ равны соответственно: $6.89,8.71$ и 7.00 эВ [28], так что средние значения $W_{d} \approx 7.5$ эВ и $\rho_{m}=10 W_{d} \approx 1.3{ } \mathrm{~B}^{-1}$. В случае сильной (ковалентной) связи получаем $\Gamma_{m} \approx 16$ эВ. Для слабой (ван-дер-ваальсовой) связи при $V=-2 D$ получаем $\Gamma_{m} \sim 10 D^{2}$, где $D$ выражено в эВ, так что по порядку величины $\Gamma \leq 1$ эВ. 
По оценкам работы [16] для СГ, сделанным с учетом экранировки кулоновского взаимодействия собственными электронами, имеем $U \approx 9.3$ и $G \approx 5.5$ эВ. Так же как и в работе [13], будем считать эти величины максимальными значениями кулоновских параметров, так как экранировка электронами металлической подложки здесь не учитывается.

\section{5. Обсуждение результатов}

Итак, мы установили, что для СГ константа $e-p h$-взаимодействия $\lambda_{\|} \sim 0.1$ эВ; для сильной и слабой связи ЭГ с подложкой $\lambda_{\perp} \sim 1$ эВ и $\lambda_{\perp}=2 D$ соответственно. Так как $\left(\lambda_{\|}+\lambda_{\perp}\right) / z G \ll 1$, отличие $G^{*}$ от $G$ мало. Таким образом, выполнение условия (10) продиктовано исключительно кулоновскими параметрами. При этом, исходя из сделанных оценок для ЭГ, в случае сильной связи неравенства (10) и (11) не выполняются, а в режиме слабой связи оба неравенства справедливы.

Согласно теории адсорбции [14], для оценки энергии квазиуровня адатома по отношению к атомному значению $\varepsilon_{0}$ помимо гибридизационного сдвига $\Lambda(\varepsilon)$ нужно учитывать кулоновский сдвиг квазиуровня $e^{2} / 4 a_{\perp}[14]$, где $e-$ заряд позитрона. Тогда вместо $\varepsilon_{0}$ получаем $\varepsilon_{0 A(B)}=\varepsilon_{0}+e^{2} / 4 a_{\perp A(B)}$, где мы предпологаем, что $a_{\perp A} \neq a_{\perp B}$. В результате в однородном по спину и заряду состоянии закон дисперсии имеет вид $E_{ \pm}^{\sigma}(\mathbf{k})= \pm \sqrt{\left(\varepsilon_{0 A}-\varepsilon_{0 B}\right)^{2} / 4+t^{2} f^{2}(\mathbf{k})}$, где функция $f(\mathbf{k})$ в точке Дирака $\mathbf{k}=0$ обращается в нуль [13]. При этом ширина щели равна

$$
\left|\varepsilon_{0 A}-\varepsilon_{0 B}\right| \approx\left(e^{2} / 4 a_{\perp}\right)\left(\delta a_{\perp} / \bar{a}_{\perp}\right),
$$

где $\bar{a}_{\perp}=\left(a_{\perp A}+a_{\perp B}\right) / 2$. Вопрос о щели в рамках модели ЕНМ достаточно подробно рассматривался в работе [13]. Полученные там результаты справедливы и для ЕННМ при условии замены $G$ на $G^{*}$.

Выше было показано, что вертикальные смещения адатомов ЭГ равны $\delta a_{\perp \alpha}=-\left(w_{\perp} / M \omega_{\perp}^{2}\right) n_{\alpha}$, так что измятость листа ЭГ графена характеризуется параметром

$$
\delta a_{\perp} \equiv\left|\delta a_{\perp A}-\delta a_{\perp B}\right| \approx 2\left|c^{\prime}\right|\left(w_{\perp} / M \omega_{\perp}^{2}\right),
$$

где штрих у $c^{\prime}=\left(n_{A}-n_{B}\right) / 2$ означает, что ненулевая разность чисел заполнения подрешеток возникла исключительно благодаря $e-p h$-взаимодействию (см. подробнее работу [15] и ссылки в ней). По данным эксперимента [29] для ЭГ на $\operatorname{Ir}(111)$ имеем $\delta a_{\perp} \approx 0.27 \AA$. При этом для слабой связи графен-подложка получаем $\left|\varepsilon_{0 A}-\varepsilon_{0 B}\right| \approx 0.07$ эВ. Следует отметить, что в работе [27] высказано предположение: если щель в спектре существует, то ее ширина должна быть меньше 0.2 эВ. О наличии узкой щели свидетельствуют также данные работ [30,31]. Следовательно, сделанные нами оценки справедливы по порядку величины, хотя для формирования щели $\sim 0.2$ эВ одного $e-p h$-взаимодействия, повидимому, недостаточно. Добавим также, что, в соответствии с данными работ [32,33] точка Дирака ЭГ на
$\operatorname{Ir}(111)$ сдвинута лишь на 0.1 эВ выше уровня Ферми, что также оправдывает наши оценки, сделанные для недопированного ЭГ.

В заключение данного раздела обратимся к модели Холстейна, предположив, что $U=G=0$. В этом случае условие (10) сводится к неравенству $\lambda>\pi \Gamma_{m} / 2$ для случая сильной связи графен-подложка, а при слабой связи - к $\lambda>t / \sqrt{z}$. Из сделанных оценок ясно, что возникновение ВЗП-состояния если и возможно, то только в последнем случае. С другой стороны, в модели ННМ $(G=0)$ неравенство (10) не выполняется, а выполнение неравенства (11) возможно только в условии слабой связи.

\section{6. Заключение}

В данной работе мы ставили целью выявить роль фононов в формировании электронного состояния ЭГ, для чего предложили расширенную модель Холстейна-Хаббарда (ЕННМ). В результате сделанных оценок и имеющихся в литературе данных показано, что для понимания влияния $e-p h$-взаимодействия на фазовые диаграммы ЭГ, полученные в работе [13] в рамках модели ЕНМ, достаточно неравенства $G<G^{*}=G+\lambda / z$. Численные оценки показали при этом, что $G \gg \lambda / z$.

Отметим, что по установившемуся в настоящее время мнению [30-33], безразмерная электрон-фонноная константа для ЭГ на переходных металлах, определенная в рамках теории Элиашберга [34], действительно мала (по крайней мере, на порядок меньше, чем в самих переходных металлах; см., однако, поправку в ссылке [32]). Тот же вывод вытекает из анализа экспериментальных данных для квазисвободного ЭГ на $\operatorname{Ir}(111)$ и $\mathrm{SiC}(0001)$ при наличии соответственно кислородного и водородного интеркалированных слоев [35]. Таким образом, в представленной в данной работе модели кулоновское взаимодействие играет ведущую роль. Следует, однако, еще раз подчеркнуть, что при этом использовались максимальные значения $U$ и $G$, полученные без учета экранировки со стороны металлического субстрата.

В модели ННМ ВЗП не возникает, а ВСП может существовать только в условии слабой связи ЭГ с подложкой. В модели Холстейна, наоборот, в условиях слабой связи может возникнуть ВЗП.

\section{Список литературы}

[1] T. Holstein. Ann. Phys., 8, 325 (1959).

[2] J. Hubbard. Proc. Royal Soc. (London), A 276, 238 (1963).

[3] M. Hohenadler, W. von der Linden. Phys. Rev. B, 71, 184309 (2005).

[4] M. Berciu. Phys. Rev. B, 75, 081101R (2007).

[5] R.P. Hardikar, R.T. Clay. Phys. Rev. B, 75, 245103 (2007).

[6] S. Kumar, J. van den Brink. Phys. Rev. B, 78, 155123 (2008).

[7] Y. Murakami, P. Werner, N. Tsuji, H. Aoki. Phys. Rev. B, 88, 125126 (2013). 
[8] M. Chakraborty, M. Tezuka, B.I. Min. Phys. Rev. B, 89, 035146 (2014).

[9] P. Werner, M. Eckstein. Europhys. Lett., 109, 37002 (2015).

[10] S. Pradhan, G.V. Pai. Phys. Rev. B, 92, 165124 (2015).

[11] I.V. Sankar, A. Chatterjee. Physica B, 489, 17 (2016).

[12] T. Miyao. arXive:1402.5202; 1610.09039.

[13] С.Ю. Давыдов. ФТТ, 59, 8 (2017).

[14] С.Ю. Давыдов. Теория адсорбции: метод модельных гамильтонианов. (СПб., Изд-во СПбГЭТУ „ЛЭТИ“, 2013).

[15] С.Ю. Давыдов. ФТТ, 55, 197 (2013).

[16] T.O. Wehling, E. Sasioglu, C. Friedrich, A.I. Lichtenstein, M.I. Katsnelson, S. Blügel. Phys. Rev. Lett., 106, 236805 (2011).

[17] С.Ю. Давыдов, О.В. Посредник. Метод связывающих орбиталей в теории полупроводников. Учеб. пособие. (СПб., Изд-во СПбГЭТУ „ЛЭТИ“, 2007). twirpx.com/file/1014608/

[18] J.-C. Charlier, P.C. Eklund, J. Zhu, A.C. Ferrari. Electron and Phonon Properties of Graphene: Their Relationship with Carbon Nanotubes. In: A. Jorio, G. Dresselhaus, M.S. Dresselhaus (eds): Carbon Nanotubes. Topics Appl. Physics, 111, 673 (2008). (Springer Verlag, Berlin-Heidelberg, 2008).

[19] K. Ishikawa, T. Ando. J. Phys. Soc. (Japan), 75, 084713 (2006).

[20] С.Ю. Давыдов, О.В. Посредник. ФТТ, 57, 819 (2015).

[21] W.A. Harrison. Phys. Rev. B, 74, 205101 (2006).

[22] Физические величины. Справочник, под ред. И.С. Григорьева, Е.3. Мейлихова (М., Энергоатомиздат, 1991).

[23] W.A. Harrison, G.K. Straub. Phys. Rev. B, 36, 2695 (1987).

[24] С.Ю. Давыдов. ФТТ, 59, 610 (2017).

[25] H. Zi-Pu, D.F. Ogletree, M.A. Van Hove, G.A. Somorjai. Surf. Sci., 180, 433 (1987).

[26] A.T. N'Diaye, S. Bleikamp, P.J. Feibelman, T. Michely. Phys. Rev. Lett., 97, 215501 (2006). Erratum: Phys. Rev. Lett., 101, 219904 (2008).

[27] Ч. Киттель. Введение в физику твердого тела. (М., Наука, 1978).

[28] В.Ю. Ирхин, Ю.П. Ирхин. Электронная структура, физические свойства и корреляционные эфбфекты в $d$ u $f$-металлах и их соединениях (Екатеринбург, УрО $\mathrm{PAH}, 2004)$.

[29] A.B. Preobrajenski, M.L. Ng, A.S. Vinogradov, N. Mårtensson. Phys. Rev. B, 78, 073401 (2008).

[30] I. Pletikosic, M. Kralj, P. Pervan, R. Brako, J. Coraux, A.T. N'Diaye, C. Busse, T. Michely. Phys. Rev. Lett., 102, 056808 (2009).

[31] R. Larciprete, S. Ulstrup, P. Lacovig, M. Dalmiglio, M. Bianchi, F. Mazzola, L. Hornekær, F. Orlando, A. Baraldi, P. Hofmann, S. Lizzit. ASC Nano, 6, 9551 (2012).

[32] S. Ulstrup, M. Bianchi, R. Hatch, D. Guan, A. Baraldi, D. Alfe, L. Hornekær, P. Hofmann. Phys. Rev. B, 86, 161402(R) (2012). Erratum: Phys. Rev. B, 93, 239901(E) (2016).

[33] M. Kralj, I. Pletikosic, M. Petrovic, P. Pervan, M. Milun, A.T. N'Diaye, C. Busse, T. Michely, J. Fujii, I. Vobornik. Phys. Rev. B, 84, 075427 (2011).

[34] Ph. Hofmann, I.Yu. Sklyadneva, E.D.L. Rienks, E.V. Chulkov. New J. Phys., 11, 125005 (2009).

[35] J.C. Johannsen, S. Ulstrup, M. Bianchi, R. Hatch, D. Guan, F. Mazzola, L. Hornekær, F. Fromm, C. Raidel, T. Seyller, P. Hofmann. J. Phys.: Condens. Matter, 25, 094001 (2013).

\section{Extended Holstein-Hubbard model for the epitaxial graphene on metal}

\author{
S.Yu. Davydov
}

loffe Physical-Technical Institute,

Russian Academy of Sciences, 194021 St. Petersburg, Russia

National Research University

of Information Technologies,

Mechanics and Optics,

197101 St. Petersburg, Russia

Abstract Model included the extended Hubbard model with intra- and inter-atomic Coulomb repulsions and the Holstein model describing interaction of the band electron with the Einstein phonon is proposed. Three regions of phase diagram are considered: spin-density wave (SDW), charge-density wave (CDW), and spin and charge uniform distribution. Numerical estimations for $\mathrm{Rh}$, Ir, and Pt substrates show that the Coulomb interactions play the leading role, making possibilities of the transitions from the unified state to the SWD and CWD states. 